\section{Tumorassoziierter Eisenmangel: Intravenöse Eisentherapie}

Das Management des tumorbedingten Eisenmangels stand im Fokus eines Symposiums auf dem Jahreskongress der Arbeitsgemeinschaft Supportive Maßnahmen in der Onkologie, Rehabilitation und Sozialmedizin der Deutschen Krebsgesellschaft (ASORS) in Berlin. Dr. Tilman Steinmetz, Köln, präsentierte aktuelle Daten einer 12-wöchigen nicht-interventionellen Studie mit 642 Tumorpatienten mit absolutem oder funktionellem Eisenmangel. Die Ergebnisse zeigen, dass eine intravenöse Therapie mit Eisencarboxymaltose (ferinject ${ }^{\oplus}$, Vifor Pharma Deutschland $\mathrm{GmbH}$ ) den Hämoglobin ( $\mathrm{Hb}$ )-Wert normalisiert. Die Wirksamkeitsanalyse schloss 420 Patienten ein. 347 Patienten hatten ausschließlich Eisencarboxymaltose erhalten, 73 Patienten zusätzlich auch Erythropoese-stimulierende Agenzien (ESAs). Der durchschnittliche Hb-Wert stieg von 9,8g/dl auf $11,3 \mathrm{~g} / \mathrm{dl}$ nach elf Wochen. Der FerritinWert verdoppelte sich von 399,2ng/ml zu Therapiebeginn auf $801,7 \mathrm{ng} / \mathrm{ml}$ zu Studienende. Die Transferrinsättigung stieg von initial $17,99 \%$ auf $33,86 \%$. Es gab keinen relevanten Unterschied im Verlauf des Hb-Wert-Anstiegs bei Patienten, die nur Eisencarboxymaltose erhielten, und Patienten, die parallel auch ESAs bekamen. Der stärkste Hb-Anstieg wurde bei Patienten mit einem Ausgangswert von $<10 \mathrm{~g} / \mathrm{dl}$ beobachtet.

\section{Miconazol-Buccaltablette bei oralen Candidosen}

Für die Therapie oraler Candidosen ist mit Loramyc ${ }^{\circledast}$ (Therabel Pharma Deutschland $\mathrm{GmbH}$, ribosepharm division Hikma Pharma GmbH) eine innovative mucoadhäsive Miconazol-Buccaltablette mit breitem Wirkspektrum gegen alle relevanten Candida-Spezies - die hauptsächlichen Verursacher oraler Candidosen - verfügbar. Die Buccaltablette enthält 50mg Miconazol und wird einmal täglich angewendet. Es wird lang anhaltend eine wirksame Miconazol-Konzentration im Speichel erreicht.

\title{
Myelodysplastische Syndrome
}

\section{Auch ältere Patienten können von Azacitidin profitieren}

\begin{abstract}
Azacitidin ist eine wesentliche Therapieoption bei älteren Patienten mit myelodysplastischen Syndromen (MDS) der IPSS-Risikogruppen Intermediär-2 und Hochrisiko. Um die Therapieentscheidung bei älteren Patienten zu erleichtern, werden Kriterien benötigt, mit denen beurteilt werden kann, inwieweit der Schweregrad vorliegender Komorbiditäten den Behandlungserfolg mit Azacitidin beeinflusst.
\end{abstract}

Sanna A et al. untersuchten dies in einer Analyse mit 127 MDS-Patienten der IPSS-Risikogruppen Intermediär-1 und -2 sowie Hochrisiko, von denen ca. ein Drittel $\geq 75$ Jahre war (Sanna A et al., 2011, EHA:\#1059, oral presentation). Die Patienten erhielten Azacitidin (Vidaza ${ }^{\circledast}$ ) in einer Dosierung von $75 \mathrm{mg} / \mathrm{m}^{2} \mathrm{KOF}$ an den Tagen 1 bis 7 jedes 28-Tage-Zyklus. Der Schweregrad der Komorbiditäten wurde parallel mit folgenden Scores bestimmt: Charlson Comorbidity Index (CCl), Cumulative Illness Rating Scale (CIRS), Adult Comor- bidity Evaluation-27 (ACE-27) und MDS-Comorbidity-Index (MDS-Cl). Es zeigte sich, dass das Ansprechen auf Azacitidin in Bezug auf alle Scores unabhängig vom jeweiligen Schweregrad der Komorbiditäten war. Das Gesamtüberleben war jedoch entscheidend mit dem Schweregrad der Komorbiditäten assoziiert.

\section{ACE-27-Score für die prognostische Evaluation}

Sanna et al. folgerten, dass eine objektivierte Erfassung der Komorbiditäten dazu beitragen kann, unter den älteren Patienten mit Komorbiditäten, diejenigen zu identifizieren, die von einer Azacitidin-Therapie profitieren. Dies scheine auf die meisten Patienten zuzutreffen. Am besten geeignet für die prognostische Evaluation einer Behandlung sei der ACE-27-Score, da sich mittels dieses Scores vier Gruppen mit deutlich unterschiedlichem Gesamtüberleben definieren ließen.

Quelle: Information der Celgene $\mathrm{GmbH}$

\section{Chemotherapie-induziertes Erbrechen Fosaprepitant-Einmalgabe kann Prophylaxe erleichtern}

Die antiemetische Prophylaxe ist ein wichtiger Bestandteil der Supportivtherapie in der Onkologie. Im Zentrum steht dabei die Lebensqualität des Patienten.

Die Dreifachkombination aus 5- $\mathrm{HT}_{3}$-Rezeptorantagonist, Neurokinin-1 (NK1)-Rezeptorantagonist und Glucocorticoid wird zur antiemetischen Prophylaxe bei hoch emetogener Chemotherapie sowie bei moderat emetogener Chemotherapie mit einem Anthrazyklin/Cyclophosphamid-Regime empfohlen (z.B. deutsche Übersetzung der MASCC/ESMO-Guidelines Antiemese 2011 unter www.onkosupport.de).

Als NK-1-Rezeptorantagonist steht neben oral zu verabreichendem Aprepitant (EMEND ${ }^{\circledR}$ ) auch intravenös zu applizierendes Fosaprepitant (IVEMEND ${ }^{\circledR}$ ) zur Verfügung. Fosaprepitant (115mg) kann im Rahmen eines 3-Tage-Schemas eingesetzt werden, seit August 2010 ist zudem die Einmalgabe von Fosaprepitant (150mg) am ersten Tag der Chemotherapie zugelassen. Die Basis der Zulassungserweiterung sind die Ergebnisse der Phase-III-Studie EASE017. Die EASE-017-Studie mit 2.322 Patienten, die erstmals eine Chemotherapie mit Cisplatin erhielten, zeigte, dass ein "All-onday-one"-Schema möglich ist, so Prof. Dr. Petra Feyer, Berlin. Die intravenöse Einmalgabe von 150mg Fosaprepitant am ersten Tag der Chemotherapie war in dieser Studie vergleichbar gut antiemetisch wirksam wie das 3-Tage-Schema mit oralem Aprepitant (primärer Endpunkt: komplettes Ansprechen - kein Erbrechen, keine Notfallmedikation für fünf Tage: 71,9\% versus 72,3\%; Grunberg SM et al., 2010, J Clin Oncol 28 [Suppl]: \#9021, poster presentation). Der NK1-Rezeptorantagonist wurde jeweils mit einem $5-\mathrm{HT}_{3}$-Rezeptorantagonisten und Dexamethason kombiniert. Unerwünschte Ereignisse waren in beiden Studienarmen vergleichbar. BEF

Quelle: Veranstaltung von u.a. MSD SHARP \& DOHME GmbH 